\title{
Kinematic Analysis of Triple Ball Tie-rod in Ackermann Steering and Tilting Mechanism for Tricycle Application
}

\author{
Wimba Pramudita Widi, Aufar Syehan, and Danardono Agus Sumarsono* \\ Department of Mechanical Engineering, Universitas Indonesia, Jalan Kampus UI, Depok, \\ Jawa Barat 16424, Indonesia
}

\begin{abstract}
Nowadays, a concept of tilting three-wheel vehicle is introduced, one of which is the electric tilting tricycle to provide an alternative mode of transportation. Some of the tilting tricycle design using a tadpole trike configuration and it needs an adequate steering system that can be synergized with tilting mechanism. The steering mechanism follows the Ackermann steering geometry. Usage of Ackermann geometry means applying a mechanism of trapezoidal four-bar linkage to the tricycle. To create and maintain the simple trapezoid shape, Triple Ball Tie-rod model, a single rod which supports three ball joints, is proposed. Since the capabilities of the model are yet to be proven, this research evaluates the usage of a tie-rod model to find out its capabilities to support the works of the steering mechanism of the tricycle. The measurements are conducted after the simulation of the 3D model to extract some data such as maximum lean angle and inner and outer steering angles. Another simulation using regular tie-rod model also conducted with the same method for comparison purposes. The results of the study are maximum allowed tilting angle and generated Ackermann steering angles. Each designed models have their respective advantages.
\end{abstract}

Keywords: Electric tadpole three wheel, misalignment, simulation of $3 \mathrm{~d}$ model, steering angle, trapezoidal four-bar linkage.

\section{Introduction}

In modern cities, excessive traffic volume is causing a traffic congestion. It is also worsened by road widening, which aims to accommodate the bigger volume of traffic causing the shortage of other public places. Moreover, traffic congestion also draws an ecological problem as air pollution is continuously being emitted.

To face those problems, narrow vehicles have studied in the last decade [1]. This kind of vehicle is absolutely smaller and lighter than regular automobiles, which can reduce the space usage of the road. Furthermore, it commonly operated by an electric powertrain, so there is no emission from the exhaust $[2,3]$. One key problem of operating the narrow vehicle is its maneuver stability, as its structure is prone to roll over because of its high

* Corresponding author: danardon@eng.ui.ac.id 
centre of gravity $[1,4]$. To overcome the problem, narrow vehicles are equipped with a tilting mechanism which allows its structure to lean just like a motorcycle does $[3,5]$ and produce advantage in cornering [6].

One of the most favoured shapes of a narrow vehicle to be studied is the three-wheeled vehicle with two wheels on the front axle [6], known as the tadpole design [5, 7]. Therefore, this study focused on the application of electric tadpole tricycle. As its front axle has two wheels, it needs an adequate steering mechanism. The steering mechanism follows the Ackermann steering, commonly used in automobiles.

Basic Ackermann steering system uses a mechanism of a four-bar linkage [8]. The shape of a simple trapezoid four-bar linkage is formed by single rigid bars joined together in the entire trapezoidal side. In the tadpole tricycle application, the steering system linkage is formed by front axle, knuckles, and tie-rod. As the most of tadpole tricycle design used two regular tie-rods, there are no longer basic four-bar linkage in their steering system because one of the trapezoid sides is formed by two bars. In order to maintain the basic shape, a tie-rod made from single rigid rod is designed. This new model has one more ball joint located in the middle of the rod in addition to the existing ball joints. Therefore, the new model is called Triple Ball Tie-rod (TBT). The middle ball joint in the TBT is designated to connect the steering arm of the tricycle and the TBT. This is expected to be a good option for a tadpole tilting tricycle steering system in the future use. This paper is written to report the initial study of this TBT model.

Since the model is applied for tilting tricycle, its capabilities to support tilting motion should be examined. The proposed examination in this paper is organized as it follows. First, the whole tricycle design -including the installed TBT model- is built using a Computer Aided Design (CAD) program. Then, tilting motion is simulated to make sure that TBT's ball joints are capable to allow the whole tricycle structure to lean. For comparison purposes, the examination is also conducted when the tricycle design is having two regular tie-rods installed. The allowance of the ball joints is determined by how far the angle of misalignment of a ball joint can reach before it collides with another part. The maximum leaning angle of the tricycle structure can be determined when one of the ball joints is about to collide. Afterward, another simulation is conducted to measure inner and outer steering angles while the angle of steering arm turns regarded as the steering input.

This paper is organized as follows. Section 2.1 explains the modelling of the tie-rod and the steering and tilting mechanism of the tricycle. Section 2.2 explains the method to estimate the maximum leaning angle of the tricycle. Then, Section 2.3 explains the steps taken to measure generated steering angles of both tie-rod models. Section 3 will show the examination result. Finally, Section 4 will conclude this paper.

\section{Design modelling and tie-rod performance examination}

\subsection{Steering and tilting mechanism 3D modelling}

\subsubsection{Tie-rod modelling}

As aforementioned, basic Ackermann steering mechanism used a mechanism of trapezoidal four-bar linkage [7]. The linkage is illustrated by a Figure 1. 


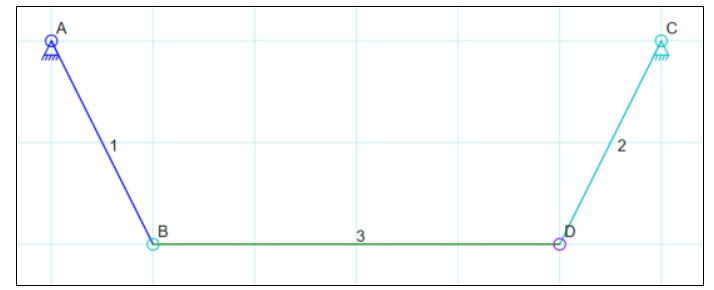

Fig. 1. Simple trapezoidal linkage used in Ackermann steering.

The main reason for making the TBT model is to form a linkage that resembled by Figure 1. The TBT itself acts as the bar number 3 that extends between B and D. To make it happen, a ball joint is made in the middle of the tie-rod as a connector to the lower steering arm since the steering system of the tricycle is not using the mechanism of rack and pinion.

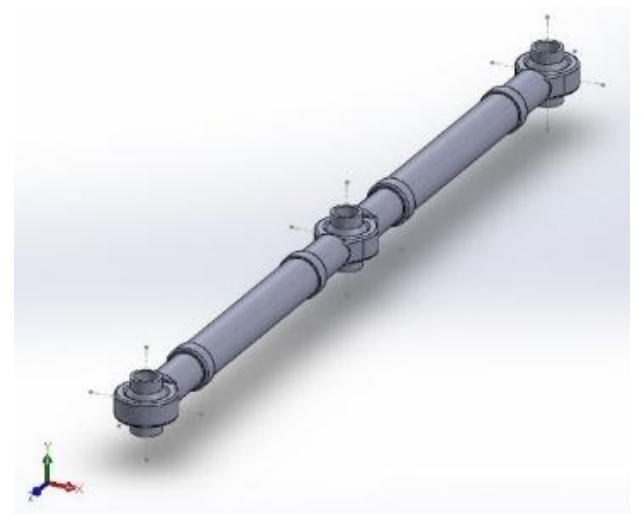

Fig. 2. Triple Ball Tie-rod 3D model.

Figure 2 is showing the 3D model of the TBT design. As can be seen, the TBT is formed by a single rigid rod. It has a ball joint at both ends to connect itself to the steering knuckles. 3D modelling of regular tie-rod also created as shown in Figure 3. The size of the ball joints on both models is made uniform with $10 \mathrm{~mm}$ hole diameter.

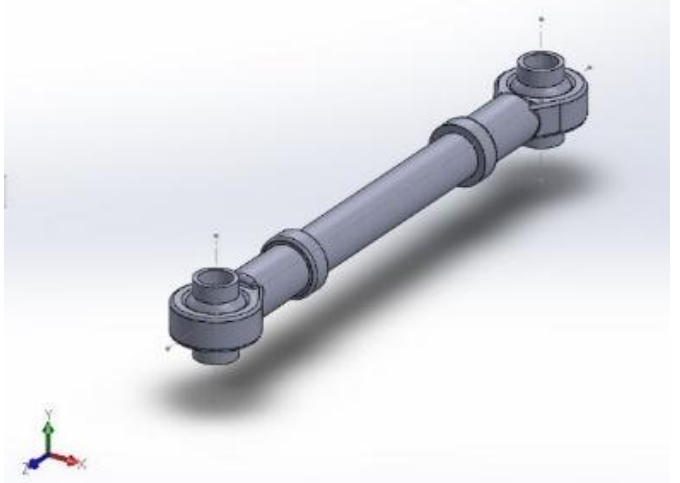

Fig. 3. Regular tie-rod 3D model.

Because of the different shapes between the two models, they also need specific steering arm design for each tie-rod model. It will be discussed further in subsection 2.1.2. 


\subsubsection{Steering system modelling}

The tricycle steering system used a simple steering system as the rider makes steering input to perform a circular motion of the steering arm. The motion of the steering arm pushes the tie-rod towards left or right.

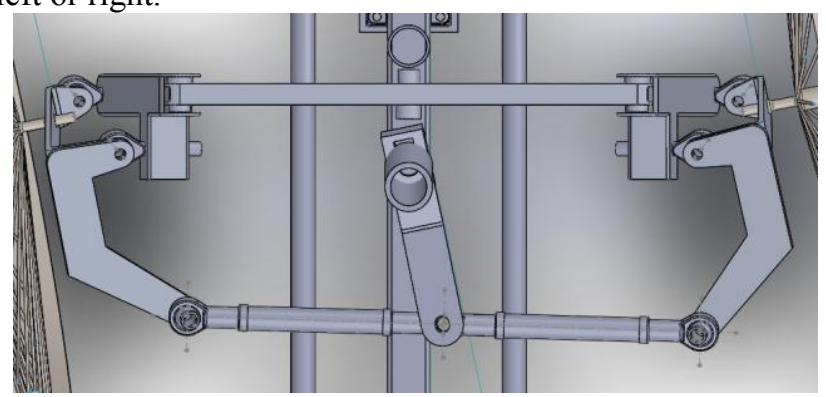

Fig. 4. Steering system using TBT model.

Figure 4 and Figure 5 illustrate the effect of the circular motion of the steering arm to the steering system. In Figure 4, steering using the TBT is being performed. As can be seen above, the shape of the trapezoidal linkage is maintained. All of the ball joints are joined to the hole of the knuckles and the steering arm. Since the middle ball joint of TBT has a straight movement to the left or right while the steering arm is having a circular movement, the hole of the steering arm is modified into a slot shape. The slot allows a relative displacement between the axis of the middle ball joint and the hole of the steering arm. The slot length is about $1 \mathrm{~mm}$ to $2 \mathrm{~mm}$.

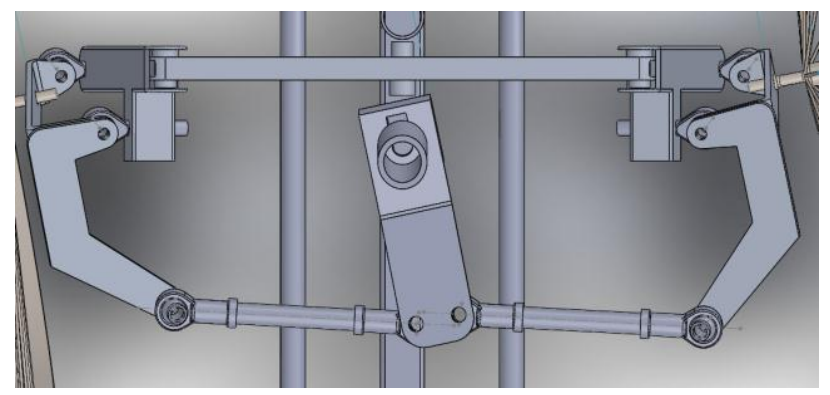

Fig. 5. Steering system using regular tie-rods.

Compared to Figure 5, while using two regular tie-rods, the trapezoid shape is altered when the system is performing the steering motion. Therefore, the B-D bar represented in Figure 1 is no more since the bar is not forming a straight line. On the other hands, the usage of two regular tie-rods needs no slot hole at the steering arm. It is because the relative displacement already occurs between the two middle ball joints. In this paper, the effects of these differences are what needs to be examined.

\subsubsection{Tilting mechanism modelling}

The tilting mechanism is built to allow the tricycle structure to lean while performing a cornering. This mechanism is formed from a parallel structure consisting of rigid bars connected to the joints. The rigid structure of the tilting mechanism is caused by the presence of a middle bar which retains the parallel arrangement of the upper and lower bars. The middle bar is illustrated by bar number 1 in Figure 6 below. The bar number 2 
and number 3 act as the tilting arms that support the structure to lean the front wheel of the tricycle. Bar number 4 and number 5 represent the upper bar.

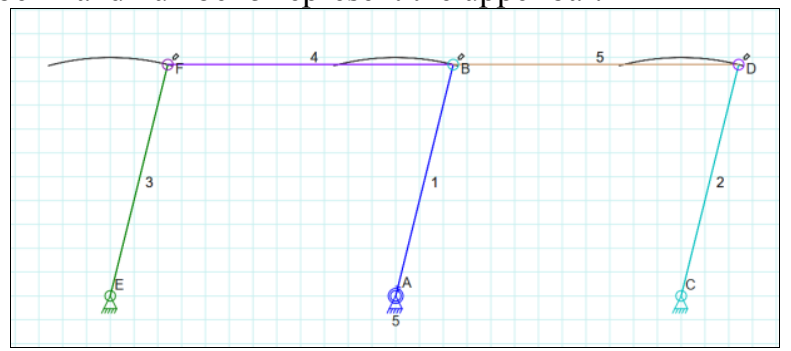

Fig. 6. Illustrations of the tilting mechanism.

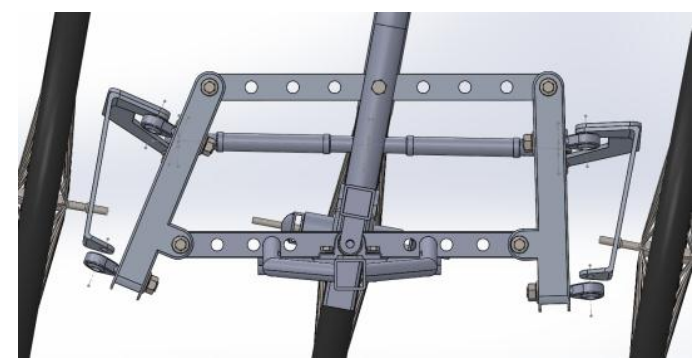

Fig. 7. Tilting mechanism in the tricycle model.

Figure 7 shows how the tilting mechanism in the tricycle works. A part of the tricycle frame acts as a middle bar to keep the parallel bars arrangement [9]. In the side of the parallel linkage, the tilting arms also designated to generate steering axis inclination while the joints to the upper and lower bar remain in a parallel arrangement.

Tilting motion of the tricycle affects the position of other parts joined with it, especially knuckles. Knuckle position will also tilt when the tricycle performs leaning motion. Tilted knuckle will affect its relative position to the ball joint of the tie-rod. Excessive lean angle will force the ball joint to collide with the end of the rod. This also needs to be examined.

\subsection{Maximum leaning angle estimation}

As aforementioned, the ball joint of the tie-rod will collide when the tricycle structure leans too much. Hence, the maximum lean angle of the model must be determined. To find the maximum lean angle, a series of steps must be done. First, the tie-rod instalment needs to be regulated. Both tie-rod models are installed at $15.85^{\circ}$ against the horizontal plane. The angle is considered as the best possible to make a large allowance of the ball joint angle of misalignment.

After the tie-rod installed in a proper manner, series of tilting motion simulated. As the simulation occurs, the examiner needs to activate the feature in to detect a collision. With the feature activated, the tricycle model cannot move further once some of its parts collide as shown in Figure 8. 


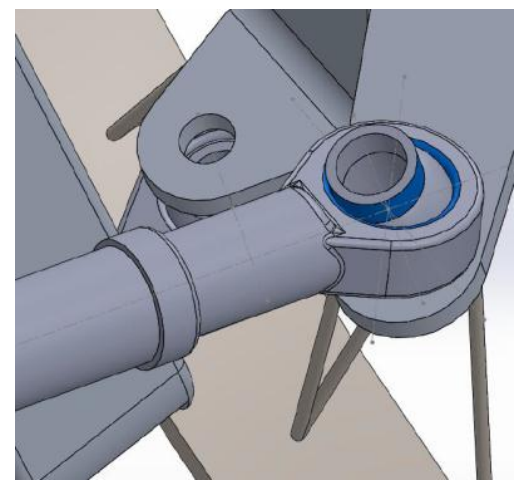

Fig. 8. Colliding surfaces in the ball joint.

As the collision happened, the position of the tricycle model is stopped at a certain lean angle. A measurement conducted to find out the ball joint angle of misalignment. The measurement method is illustrated by Figure 9. Some imaginary centrelines are sketched in the ball joint. The centrelines are drawn on the same length to resemble the radius of a ball. Then, the distance between the centreline ends is measured. This distance value resembles the length of a circle chord. Hence, the angle of misalignment can be determined as follows.

$$
\theta_{m i s}=2\left(\sin ^{-1} \frac{c}{2 C L}\right)
$$

While $\theta_{\text {mis }}$ is the angle of misalignment, $c$ is the chord length, and $C L$ is the length of the imaginary centrelines.
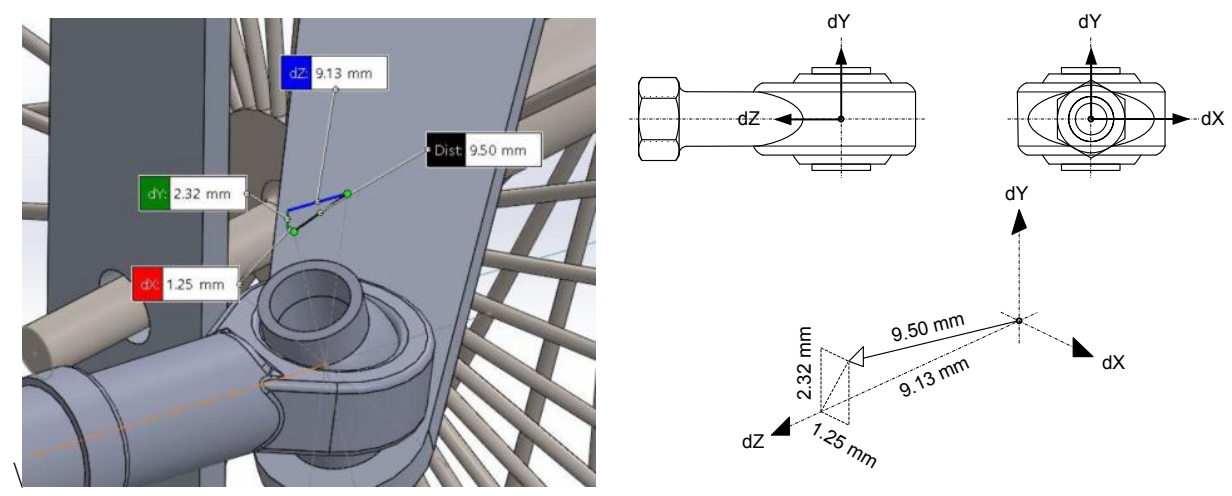

Fig. 9. Measuring the ball joint angle of misalignment.

Under the exact same position, the measurement of the tricycle leaning angle also conducted. Using dimension tool in SOLIDWORKS, relative angle can be measured between the representing surfaces. The readable number can be regarded as the value of the tricycle leaning angle. As the leaning angle is being measured while the tricycle cannot lean any further, therefore, the value can be considered as the maximum lean angle. 


\subsection{Steering angle measurement}

The next thing to be examined is the tie-rod capability to generate the steering angle. It is important to find out whether the designed TBT is generating appropriate Ackermann steering, even though TBT forms a proper four-bar linkage.

The simulation process is done by adjusting the angle of steering arm turn as the input. In this paper, the simulated input angle range is $0^{\circ}$ to $20^{\circ}$. While the steering input angle is being adjusted, the dimension tool is used to measure the steering angle of the front wheels.

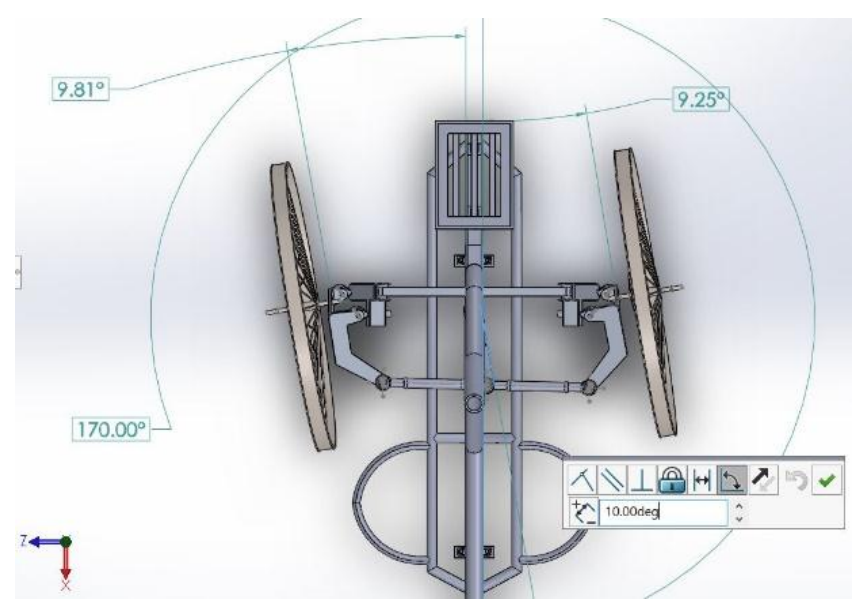

Fig. 10. Measuring the steering angles.

When the steering system is modelled correctly, the measurement should show that the inner steering angle has a greater value than the outer steering angle -as exemplified in Figure 10. The next step taken is to compare the measurement result of both of the tie-rod models to the Ackermann angles at $100 \%$ Ackermann. Ackermann steering angles can be determined as follows [10].

$$
\delta_{i, A c k}=\tan ^{-1}\left(\frac{L}{\frac{L}{\tan \delta_{o}}-t}\right)
$$

While $\delta_{i}$, Ack is the inner steering angle at $100 \%$ Ackermann, $\delta_{o}$ is the outer steering angle, $L$ and $t$ is wheelbase and track of the tricycle model, respectively. In this tricycle prototype, the value of $L$ is $1070 \mathrm{~mm}$, and the value of $t$ is $610 \mathrm{~mm}$.

\section{Examination Result}

\subsection{Maximum leaning angle}

The tie-rod has ball joints that can perform the angle of misalignment. The greater the generated value of $\theta_{\text {mis }}$, the allowance for the tricycle to lean is also greater. By using the equation (1), the value of $\theta_{\text {mis }}$ is shown in the tables below. 
Table 1. Ball joint angle of misalignment of TBT model.

\begin{tabular}{|c|c|c|c|}
\hline \multirow{2}{*}{ Position } & \multicolumn{3}{|c|}{$\boldsymbol{\theta}_{\text {mis }}$ of the ball joint (degree) } \\
\cline { 2 - 4 } & Left & Middle & Right \\
\hline Upright & 11.5935 & 14.9392 & 11.6166 \\
\hline Leaning left & 3.3924 & 15.1704 & 21.9523 \\
\hline Leaning right & 22.2792 & 15.1935 & 3.5070 \\
\hline
\end{tabular}

Table 2. Ball joint angle of misalignment of the regular tie-rod model.

\begin{tabular}{|c|c|c|c|c|}
\hline \multirow{2}{*}{ Position } & \multicolumn{4}{|c|}{$\boldsymbol{\theta}_{\text {mis }}$ of the ball joint (degree) } \\
\cline { 2 - 5 } & Left & Middle left & Middle right & Right \\
\hline Upright & 11.6166 & 14.9392 & 14.9392 & 11.6166 \\
\hline Leaning left & 3.3695 & 15.1241 & 15.1241 & 20.9259 \\
\hline Leaning right & 20.9492 & 15.1241 & 15.1241 & 3.3695 \\
\hline
\end{tabular}

Ball joint collision occurred at the side ball joint because of the relatively extreme tilt of the knuckles while the tricycle structure is leaning. As can be seen in Table 1 and Table 2, the angle value which exceed of $20^{\circ}$ is the ball joint angle of misalignment when the collision occurred at it. The allowance can be determined by the angle at collision is minus by the value at upright position, the result is as follows;

- TBT model, left lean $\quad=\left(21.9523^{\circ}-11.6166^{\circ}\right)=10.3357^{\circ}$

- TBT model, right lean $\quad=\left(22.2792^{\circ}-11.5935^{\circ}\right)=10.6857^{\circ}$

- Regular tie-rod, left lean $=\left(20.9259^{\circ}-11.6166^{\circ}\right)=9.3093^{\circ}$

- Regular tie-rod, right lean $=\left(20.9492^{\circ}-11.6166^{\circ}\right)=9.3326^{\circ}$

The TBT instalment is giving more allowance than the regular counterpart. Therefore, the usage of the TBT allows greater lean angle. The maximum lean angle value retrieved from the Table 3 .

Table 3. Ball joint angle of misalignment of TBT model.

\begin{tabular}{|c|c|c|}
\hline \multirow{2}{*}{ Lean direction } & \multicolumn{2}{|c|}{ Lean angle (degree) } \\
\cline { 2 - 3 } & TBT & Regular tie-rod \\
\hline Left & 11.69 & 9.74 \\
\hline Right & 12.05 & 9.76 \\
\hline
\end{tabular}

\subsection{Steering angles}

The measurement result of both of the tie-rod models compared to the $100 \%$ Ackerman angles is shown in Figure 11 and Figure 12 as follows. 


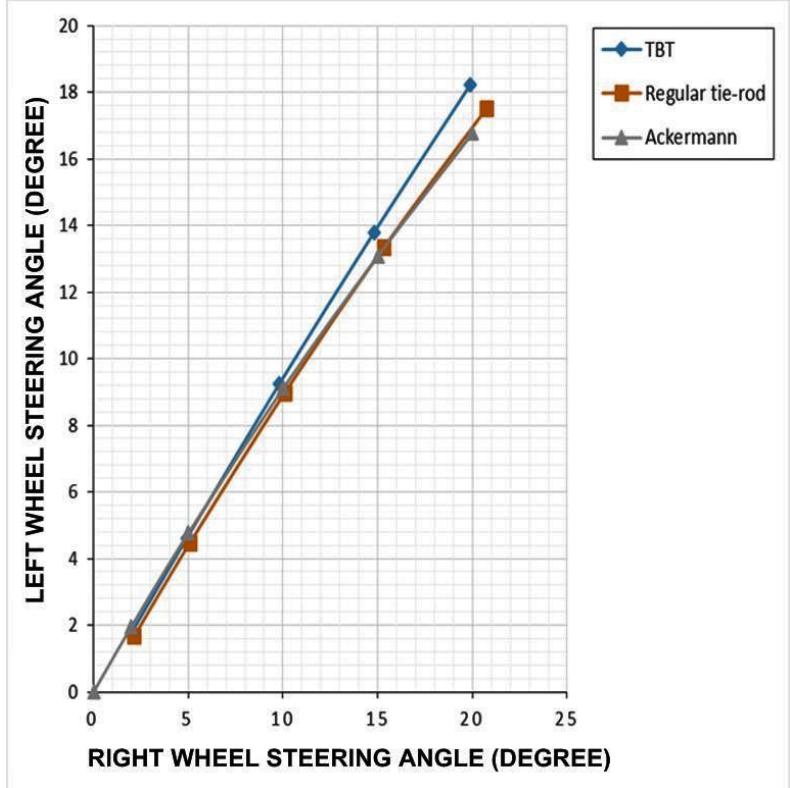

Fig. 11. Steering angle performance.

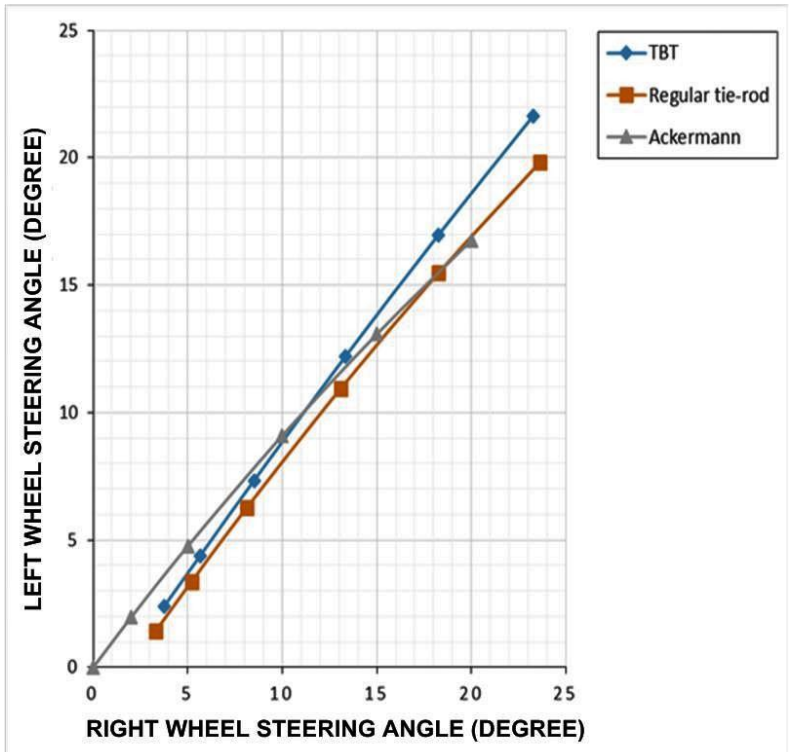

Fig. 12. Steering angle performance while leaning.

The graphs above are the result of the plotting of left wheel steering angle respect to the right wheel steering angle. In Figure 11, the data on the graph is taken at the upright position while in Figure 12. the data on the graph is taken at the leaning position. The graph for the Ackerman angles remains in standard position. The graph also shows that the leaning position of the tricycle can increase the turning performance since the steering angle of the wheel is greater than turning in the upright position. 
From the graphs above, it could be said that the usage of the regular tie-rod model is still closer to the $100 \%$ Ackermann steering performance. In response to this result, the design of the linkage is predicted as a strong factor. As explained in Subsection 2.1.2, each tie-rod model needs a specific steering arm. The shape and position of the steering arm hole could affect the position, movement, and displacement of the tie-rod so that the knuckles rotated about the different angle. Therefore, as the new model, the dimensions of the TBT and its linkage design is still needed to be evaluated further to generate the proper Ackermann steering angles. A further experiment also needs to be done by doing a tilting tricycle prototype test ride with the manufactured TBT installed.

\section{Conclusions}

In this paper, TBT is designed to form a proper four-bar linkage for the Ackermann steering mechanism in the tricycle. In order to find the proof, modelling of regular tie-rod linkage also made as comparison during the series of examination. As an initial study, this study uses the motion simulation of tie-rod 3D models. Using a tilting tricycle 3D model, the capability of TBT and regular tie-rod model to support leaning motion and generating Ackermann steering angle needs to be examined. Each tie-rod model needs a specific steering arm to connect it to the steering shaft. The steering mechanism leaning and steering motion is tested for the further measurement of the values of maximum leaning angle and steering angles. As the result, the usage of TBT allows greater leaning angle, on the other hand, the usage of regular tie-rod is better in terms of reaching closer to the $100 \%$ Ackermann.

\section{Acknowledgment}

This work is supported by Hibah PITTA 2018 (Publikasi Internasional Terindeks untuk Tugas Akhir). Funded by DRPM Universitas Indonesia (Contract Letter Number: 5000/UN2.R3.1/HKP.05.00/2018).

\section{References}

1. U. Wasiwitono, I.N. Sutantra, Yohanes, Y. Triwinarno. Applied Mechanics and Materials, 758:173-178(2015). https://www.scientific.net/AMM.758.173

2. K. Jeong, S. Choi. Road bank angle estimation for three wheel tilting vehicle using multi model estimator. The 2nd International Conference on Mechanical, Aeronautical and Automotive Engineering (ICMAA 2018) (Singapore, 2018). MATEC Web of Conferences 166,02008(2018). https://www.matecconferences.org/articles/matecconf/abs/2018/25/matecconf_icmaa2018 02008/matecc onf icmaa2018 02008.html

3. J.W. Robertson, J. Darling, A.R. Plummer. Proceedings of the Institution of Mechanical Engineers Part D: Journal of Automobile Engineering, 228,8:847862(2014). https://researchportal.bath.ac.uk/en/publications/combined-steering-anddirect-tilt-control-for-the-enhancement-of-

4. J.H. Berote. Dynamics and control of a tilting three wheeed vehicle. [Thesis]. University of Bath, Bath, UK (2010). p. 9.

https://researchportal.bath.ac.uk/en/studentTheses/dynamics-and-control-of-a-tiltingthree-wheeled-vehicle 
5. A.M. Mane, J.M. Salunkhe, A.S. Chorge, A. Muragonde, P. Bamankar. International Engineering Research Journal (IERJ), Special Issue:102-104(2017).

https://www.academia.edu/32995024/Design_and_fabrication_of universal tilting thr ee wheeler mechanism.

6. M.A. Saeedi, R. Kazemi. Int. J. Automotive Eng., 3,1:343-355(2013). https://www.researchgate.net/publication/248398794_Stability_of_Three-

Wheeled Vehicles with and without Control System

7. A. Zandieh. Dynamics of a three-wheel vehicle with tadpole design. [Master Thesis]. University of Waterloo, Ontario, Canada (2014). p. 1.

https://uwspace.uwaterloo.ca/handle/10012/9071

8. J.S Zhao, X. Liu, Z.J. Feng, J.S. Dai. Proceedings of the Institution of Mechanical Engineers Part C: Journal of Mechanical Engineering Science, 227,11:25492562(2013). https://journals.sagepub.com/doi/10.1177/0954406213475980

9. S. Nurse, R. Napper, M. Richardson. The evolution of cycles from front wheel drive to delta tilting trike. Australasian Transport Research Forum, (Melbourne, Australia, 2016). https://www.researchgate.net/publication $/ 310673140$

10. A.R. Kumar, S. Jagan, K.M. Prakash, S. Jeyabalamurugan, M.K. Hariharan, Optimization of steering geometry by design and analysis. $5^{\text {th }}$ National Conference on Trends in Automotive Parts Systems and Applications, (Tamilnadu, India, 2017). International Journal of Innovative Research in Science, Engineering, and Technology, 6,7:7-12(2017). https://www.ijirset.com/upload/2017/tapsa/2_auto_002.PDF 